\title{
Randomness of Polymer Microstructure in the Resist Film as Shot Noise
}

\author{
Makoto Muramatsu $^{1 *}$, Arisa Hara ${ }^{2}$, Satoru Shimura ${ }^{2}$, and Hidetami Yaegashi ${ }^{3}$ \\ ${ }^{1}$ Advanced Technology Development Department, Tokyo Electron Kyushu Ltd., \\ 1-1 Fukuhara, Koshi City, Kumamoto 861-1116, Japan \\ 2 Process Technology Department, Tokyo Electron Kyushu Ltd., \\ 1-1 Fukuhara, Koshi City, Kumamoto 861-1116, Japan \\ ${ }^{3}$ TEL Corporate R\&D, Tokyo Electron limited, \\ 3-1 Akasaka 5-chome, Minato-ku, Tokyo 107-6325, Japan \\ *makoto.muramatsu@tel.com
}

\begin{abstract}
Extreme ultraviolet (EUV) with a wavelength of $13.5 \mathrm{~nm}$ has already been mass-produced, but serious technical problems remain an important issue. One of them is the line edge roughness (LER) value for critical dimension (CD). Local variations such as intra-filed CD uniformity and LER are usually identified from the average CD calculated using top-down view observations. However, it is not possible to get an overall picture of LER. In this study, cross-section SEM was applied efficiently to visualize or quantify the basic behavior of the resist. In addition, the resolution limits and minimum structural units that make up the resist pattern associated with LER are discussed.
\end{abstract}

Keywords: Stochastics, Polymer dynamics, Polymer aggregate, LER, missing defect, EUV, Resolution limit

\section{Introduction}

It is aggressively discussed that the occurrence of stochastic print failures is an important factor to consider. While two main types of shot noise [1], photons and acids, are generally focused, the backbone polymer of resist materials is less considered, but cannot be negligible. Some random phenomena, as shown in Figure 1, were not first discovered in the EUV era, but before the $248 \mathrm{~nm}$ era at the latest. Therefore, the question is whether such randomness is an EUV-specific phenomenon.

In the practical region of the focus exposure matrix (FEM) to derive mean $\mathrm{CD}$, the resist pattern profile is relatively stable, and its randomness is minimal. Therefore, resist pattern profile in the early state with extremely lower dose conditions using negative tone development (NTD) type resist were observed. The main purpose of this observation is to determine the smallest structural unit of the resist pattern. And another important point is to understand the interaction reaction between the resist film and the underlying layer to find a specific solution for defect reduction.

In This paper, assumptions about the main factors of LER formation and the resolution limits of EUV resists is introduced and mentions important messages of defect induction.

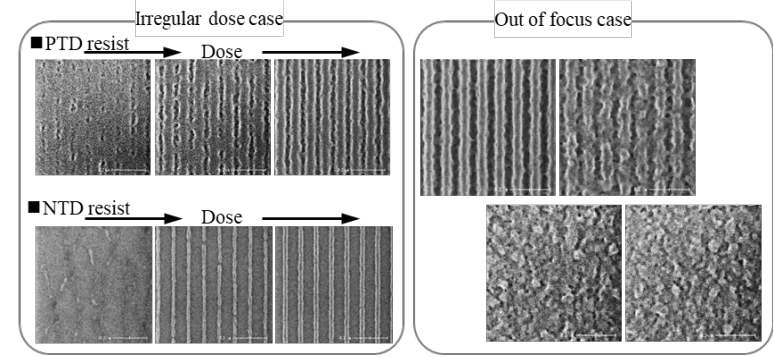

Fig. 1. Various randomness in resist patterning under non optimized condition.

\section{Experimental Results and Discussion}

2.1. Observation under extreme lower dose

The resist dissolution contrast curve, like FEM, is an important source for easily predicting the basic performance of some lithography. Although 
resist pattern profiles can be derived from contrast curves, it is not suitable for more equitable detection of stochastic processes such as resist residues in the resist open space region. In order to accurately observe the microscopic resist behavior, the surface of the contrast curve sample was observed and visualized by cross-section SEM. In this study, NTD type resist [2], which is a negative tone image, was used mainly because it has easier characteristics than a positive tone development (PTD) type resist. Because this NTD type resist was a $193 \mathrm{~nm}$ photosensitive material, the $193 \mathrm{~nm}$ scanner was selected for this test. The focal region to be visualized was the low dose state before reaching Eth, and the NTD resist film growth process with exposure was characterized by AFM and cross-section SEM images. First, the results of AFM are introduced in Fig. 2 [3-4].

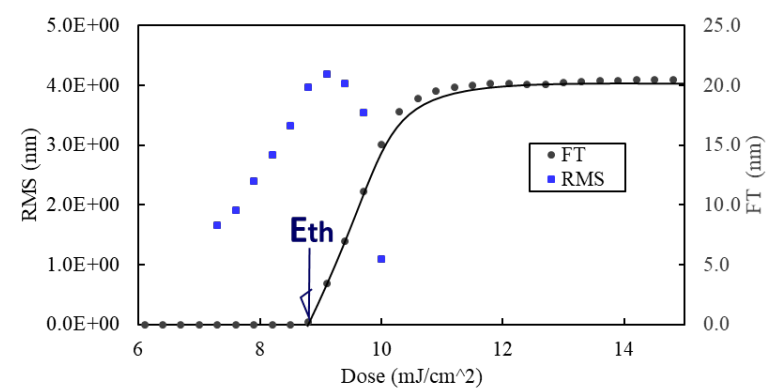

Fig. 2. Roughness trend through exposure does until Eth.

The RMS of surface roughness generated on the substrate increased with the exposure energy up to the Eth point. There may be small objects on the substrate as a resist film that has been photo-reacted and insolubilized. When the visualization results of the insoluble matter are summarized in Table 1, this growth behavior was steadily increasing with small particles generated in some places. Then it was connected in a chain reaction and finally it was filled on the substrate.

Table 1. Polymer aggregates image on under layer surface through dose.
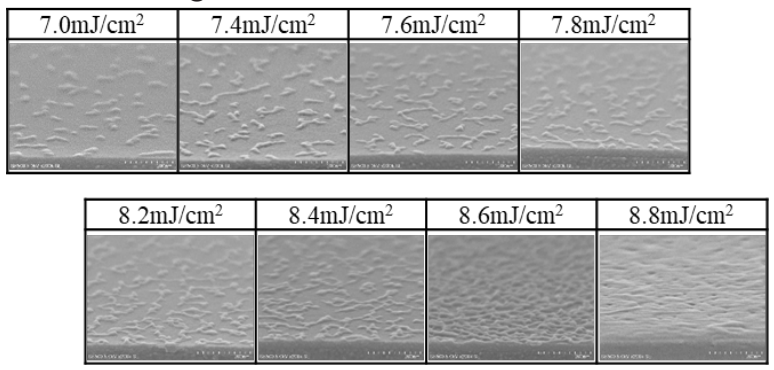

The coverage of the polymer particles on the wafer was plotted in Figure 3 to confirm that the resist film thickness measurement started with a coating point simulation. This point which became thickness measurable corresponds to Eth. Such behavioral analytics must be very important given the specific solutions for defect suppression. Because this test was a macroscopic method, observations of smaller areas under pattern exposure were made.

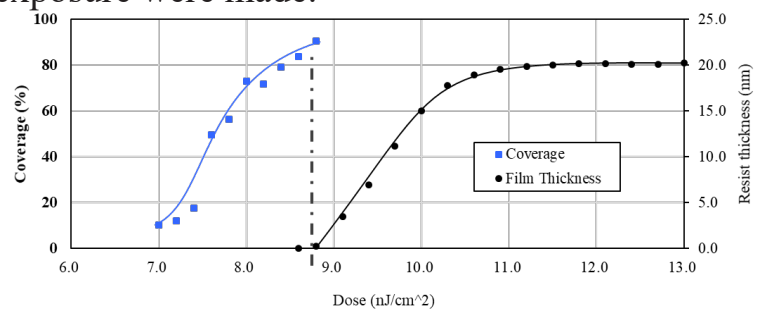

Fig. 3. Coverage trend of polymer aggregates over substrate.

\subsection{Preliminary state observation of resist pattern}

As the continuous work, the behavior of the resist in the pattern formation during the patterning exposure process step was visualized. The CD trend from patterning to nominal dose is plotted in Figure 4. Since this CD trend has three different phases, the behaviors in each phase were individually characterized.

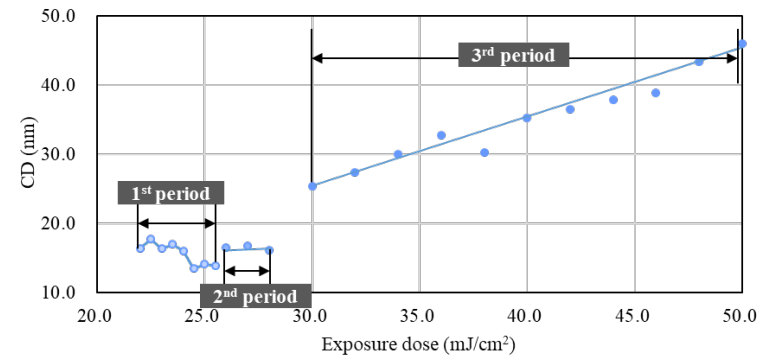

Fig. 4. Resist pattern creation behavior divided in three-periods.

$1^{\text {st }}$ period

As shown in Table 2, the generation of the particulate dotted pattern on the substrate is first captured. Since the particles have a weak adhesive force to the substrate, they partially adhered and partially floated.

Table 2. Particulate dotted pattern SEM image in preliminary state at minimum exposure dose.
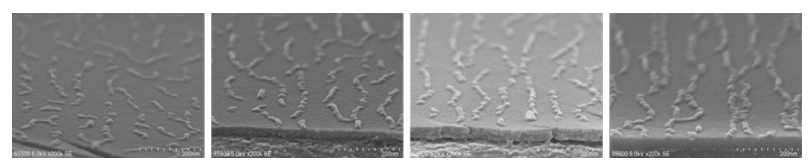
The CD size at this period is about $12 \mathrm{~nm}$, suggesting that it is the smallest structural unit that construct the resist pattern.

\section{- $2^{\text {nd }}$ period}

During this period, the dots are gathered and connected mutually. It looks like a line pattern; however, the adhesive strength is not strong enough and the pattern peeled off in spots. The average $\mathrm{CD}$ is kept constant at about $12 \mathrm{~nm}$.

Table 3. Transition state SEM image before enough adhesive force implementation.
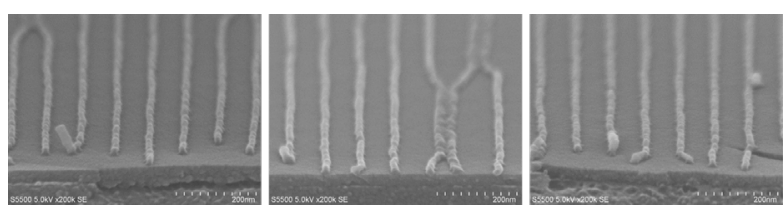

$3^{\text {rd }}$ period

The resist pattern is getting stable and the pattern is growing efficiently. The 3 sigma of CD deviation is also stable, and from this phase, the resist pattern can be visualized by CD-SEM and the $\mathrm{CD}$ can be measured.

Table 4. Resist line pattern image on SEM in steady state.

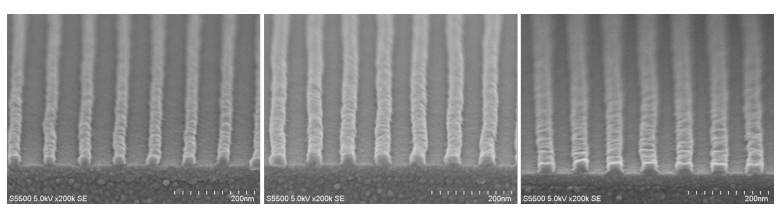

Considering this particle observation work comprehensively, a resist pattern began to be formed from the size of $12 \mathrm{~nm}$ in the first phase. The size is assumed to be the smallest structural unit for forming a resist pattern, and the resolution limit of EUV resists should be limited to about $12 \mathrm{~nm}$. Another notable result is the slight appearance of pixelated textures in the resist pattern, as shown in Table 5. The resist pattern seems consists of this $12 \mathrm{~nm}$ size structural unit.

Table 5. Top-down image of pixelated elemental unit constructing resist pattern.

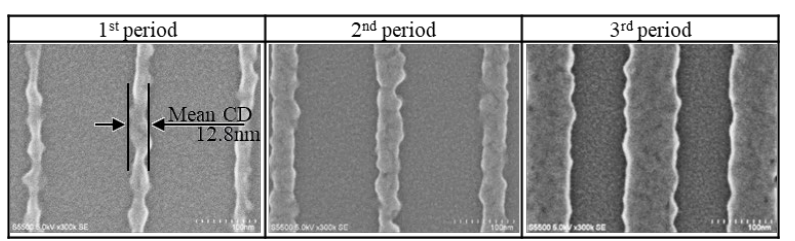

\subsection{Structural elemental unit of resist film}

This section presents the visualization results of polymer aggregates in resist membranes. Assuming the resist pattern is constructed of agglomerated polymer, the carbon density in the space of the polymer agglomerates will be significantly lower. Therefore, removing the part with low carbon density using argon sputtering was tested. With newly specializing spattering condition [5-7], the low-density part was removed successfully, and an object like a random fingerprint appeared (Figure 5).

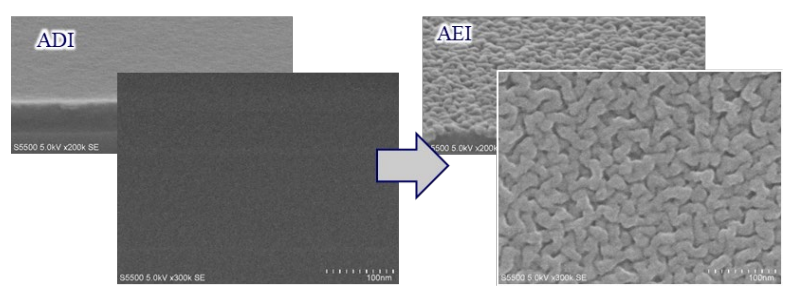

Fig. 5. Successful visualization of linear carbon cluster latent in resist film.

It looks like a fingerprint pattern using Directed Self-Assembly (DSA). When the line width was measured, the average value was $12.6 \mathrm{~nm}$. As shown in Figure 6, the kurtosis of the CD distribution plot is sharp. This special sputtering method also allows carbon agglomerates to float in the tight spaces of a patterned sample, as shown in Figure 7. Interestingly, the winding shape of the LER matches the contour of the linear carbon cluster. Therefore, this cluster can be one of the decisive factors in generating LER and should be minimized.

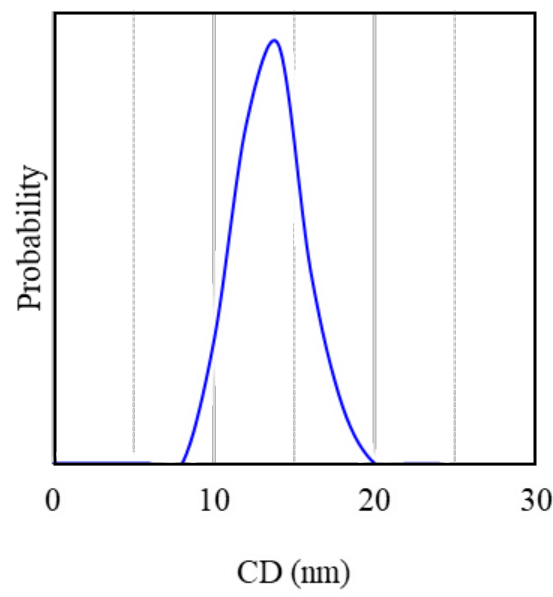

Fig. 6. Line width distribution of carbon cluster. 


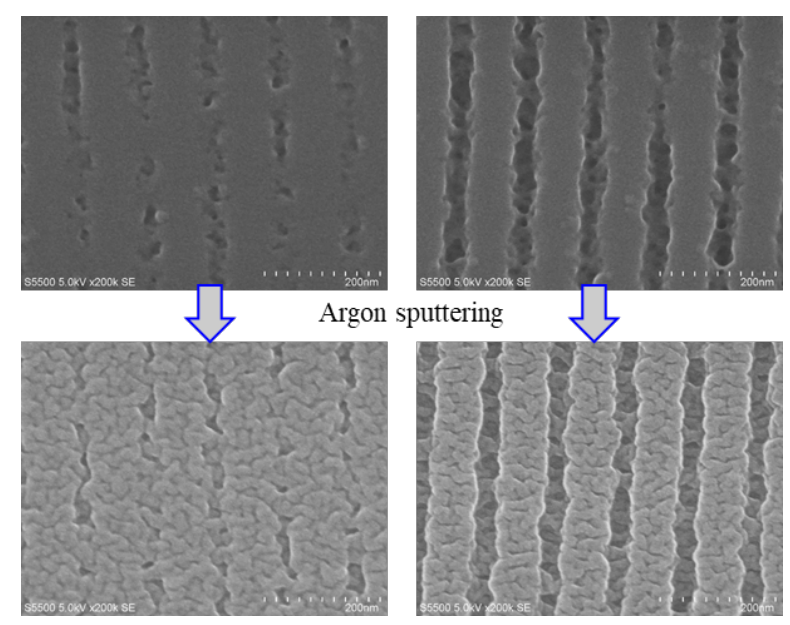

Fig. 7. Carbon cluster appearance on pattern resist sample.

\subsection{Exploration of carbon aggregates control factor}

This section considers suitable control factors in three technical areas: photochemical reactions, track processes, and resist material design. In this study, a special EUV resist sensitive to $193 \mathrm{~nm}$ was used in a $193 \mathrm{~nm}$ exposure experimental environment.
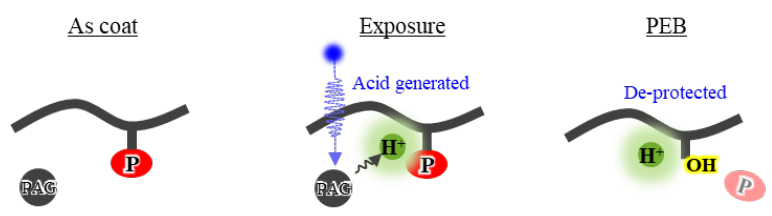

Fig. 8. Simple scheme for the photochemical reaction of chemically amplified resist process.

As shown in the schematic diagram of Figure 8, decomposition occurs by the chemical amplification reaction by UV irradiation and the thermal reaction of the acid in the resist film [8]. The line width of the linear carbon cluster and its distribution were compared at each process step, and the comparison results are shown in Table 6.

Table 6. Variability test result in photo-chemical reaction: appearance, mean $\mathrm{CD}$.

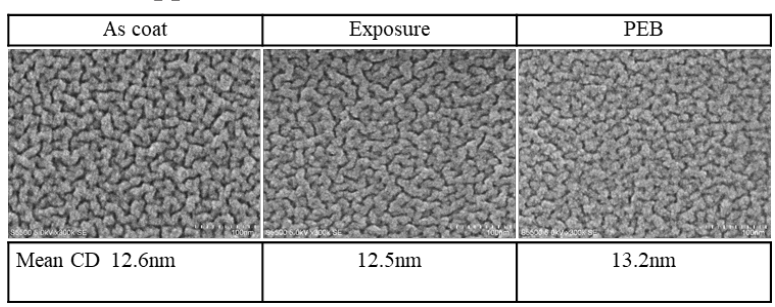

The appearance of the SEM image at each step did not change, and the $\mathrm{CD}$ value did not change at all. This result provides one piece of evidence that the chemical reaction is not involved in polymer agglutination. Further checks were made on the association of PAG with aggregation by comparing the two types of resists.

One was a nominal resist material and the other was a PAG unmixed test sample. As a result, there was no difference in the appearance of the SEM and the comparison of the average CD value (Figure 9). It can be said that the aggregate is composed only of polymer and has nothing to do with other additives such as PAG.
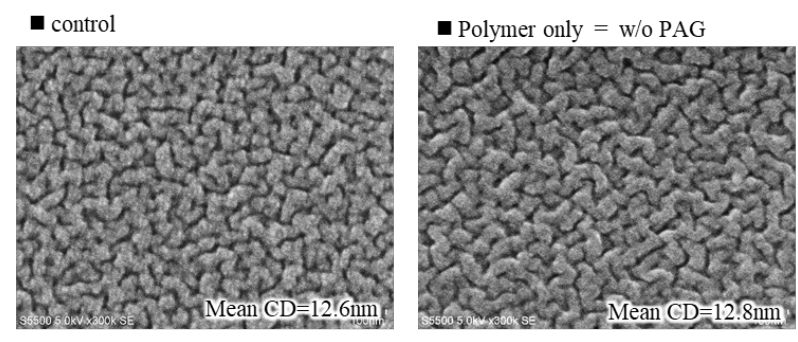

Fig. 9. Variability test result in material composition: nominal resist and polymer without PAG.

Next, the relationship with the track process factor was investigated. Given the conformation of the polymer, in the liquid or solid phase, the stable state is different for each phase. The resist drying and solvent volatilization mechanisms are important in determining the polymer conformation. Comparing the three types of resist drying, nominal spin drying and soft baking, spin drying only and natural drying, no clear difference was found in appearance, texture randomness, or average CD (Figure 10).

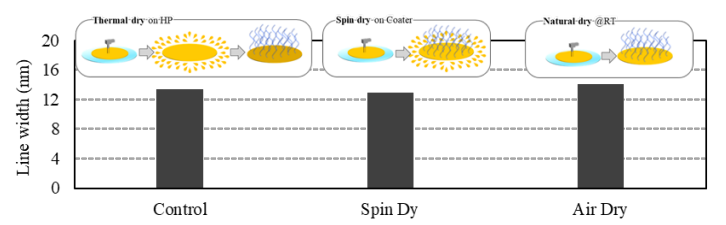

Fig. 10. Variability test result in various resist volatilization method.

According to a paper by Professor Tanaka of Kyushu University, the conformation of the polymer was changed by centrifugal force in the spin casting method as compared with the solvent casting method. The polymer conformation was confirmed throughout the wafer because the stress resisting the film was different at each point throughout the wafer due to centrifugal force. The centrifugal force at the center of the wafer is "0", which is $22.2 \mathrm{~N}(2.26 \mathrm{kgf})$ at the 
edge position of the $300 \mathrm{~mm}$ wafer. The random texture of the polymer aggregate unexpectedly remained in its original condition at each test point (Figure 11). From the same point of view, the relaxation effect of the polymer by thermal annealing was investigated, however none were changed (Figure. 12). According to these test results related to the track process, it must be said that resist tracks do not have effective control over polymer aggregate.
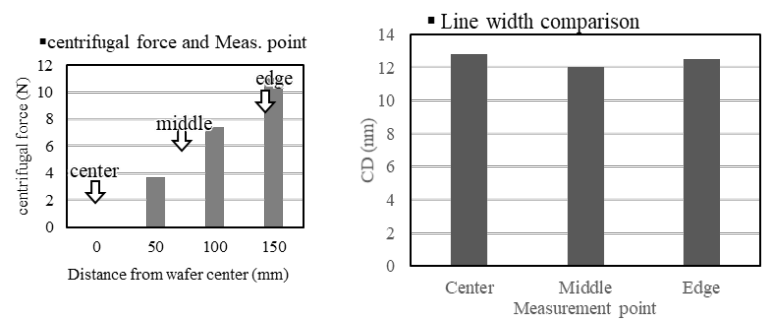

Fig. 11. Examination result of stress impact in resist film formation.

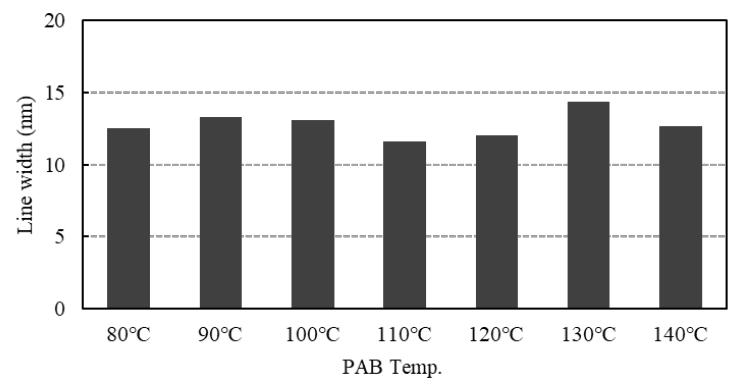

Fig. 12. Annealing effect test result for film stress mitigation related polymer conformation.

The material design of the resist is also important for investigating the conformation of the polymer. In general, EUV backbone polymers are composed of two types of units, an acrylate unit and a PHS (polyhydrostyrene), and the compound ratio is generally optimized by resist synthesis. Therefore, materials with different compound ratios were investigated. One test resist is Acrylate-rich material and other one is PHS-rich type. Obvious mean line width difference, $12.8 \mathrm{~nm}$ and $10.9 \mathrm{~nm}$ was found in this comparison (Figure 13). Basically, molecular weight is optimized for downward scaling and EUV resist has smaller molecular weight of 193 resist in conventional practice. It seemed that line width of carbon linear cluster was getting smaller in the case of lower molecular weight. In this case, EUV resist had a half molecular weight and then line width varied from $12.6 \mathrm{~nm}$ to $9.8 \mathrm{~nm}$ (Figure 14).
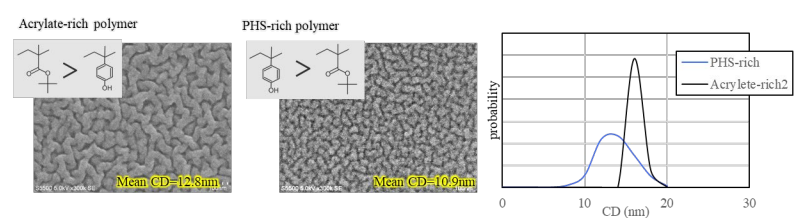

Fig. 13. Variation check result in polymer design in resist material: Acrylate-PHS combination.
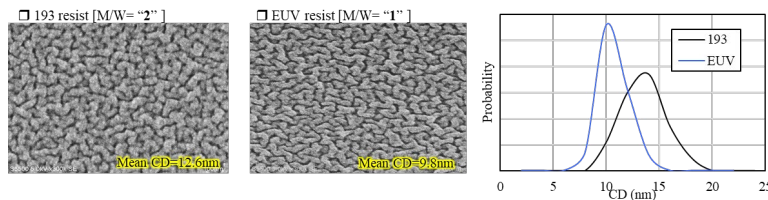

Fig. 14. Variation check result in polymer design in resist material: Molecular weight modification.

The results in this section show that the line width of linear carbon clusters can be changed by minimizing polymer design. Polymer design may be more important than a description of photon and PAG behavior.

\subsection{Depth profile of polymer grain of resist film}

According to the previous section, there are three important things to reduce LER.

$>$ Resist film construct with linear carbon cluster

$>$ Winding shape of LER match the contour of carbon cluster

$>$ Line width of cluster strongly depends on polymer molecular weight

Since the molecular weight of EUV resist is lower than that of $193 \mathrm{~nm}$ resist, the LER of EUV resist should be small. But on the contrary, the LER is worse than $193 \mathrm{~nm}$. Therefore, it should be recognized that another potential factor influences the increase in LER. First, the thickness of EUV resist is thinner than that of 193 $\mathrm{nm}$ due to its continuous scaling. The nominal resist thickness in the $193 \mathrm{~nm}$ era (about $70 \mathrm{~nm}$ ) was reduced to less than $20 \mathrm{~nm}$ in the EUV era to prevent pattern collapse.

Now, when the resist film is made extremely thin, a particularly interesting phenomenon occurs. That is, the top surface of the resist pattern becomes rough. Based on this result, the depth profile of the carbon cluster was confirmed. For the purpose, various thicknesses of resist films were prepared in the same way as the contrast curve was made, and then the samples were slightly etched with argon plasma as 
described above. The appearance of the carbon clusters at each slice level is shown in Figure 15 and the line widths are plotted in Figure 16.
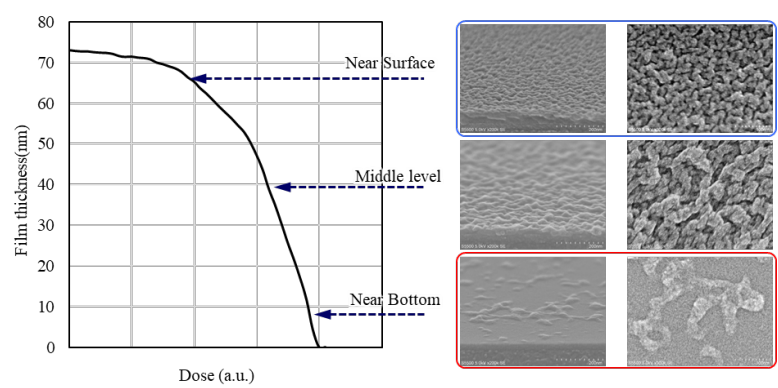

Fig. 15. Depth profile of carbon cluster appearance through resist film.

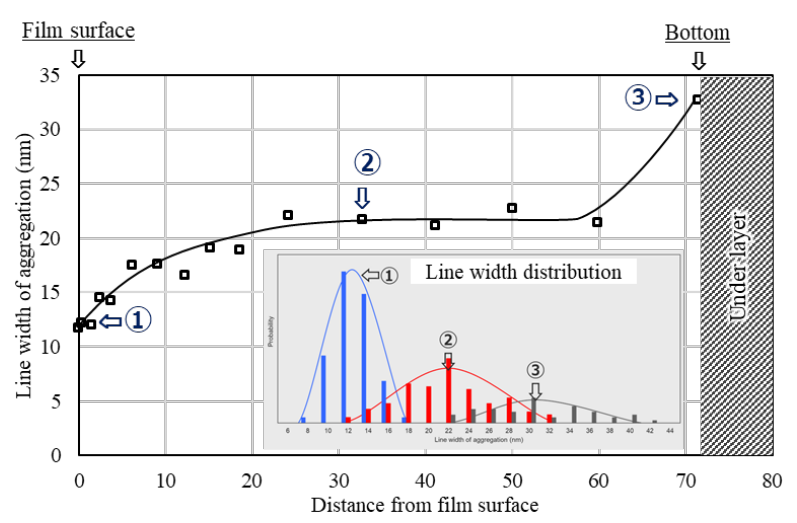

Fig. 16. CD variation in each slice level through resist film.

Line width variation is,

- Near surface: smallest, around $12 \mathrm{~nm}$,

- Bulk area: getting bigger to $22 \mathrm{~nm}$,

- Near bottom: getting bigger more to $33 \mathrm{~nm}$.

As summarized in Figure 17 [9], the significant increase in roughness when thinning the resist can be due to the larger sized carbon clusters formed near the bottom of the resist.

Simply put

resist film was thinned @EUV

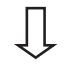

bigger aggregates at bottom

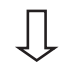

bigger LER identified eventually

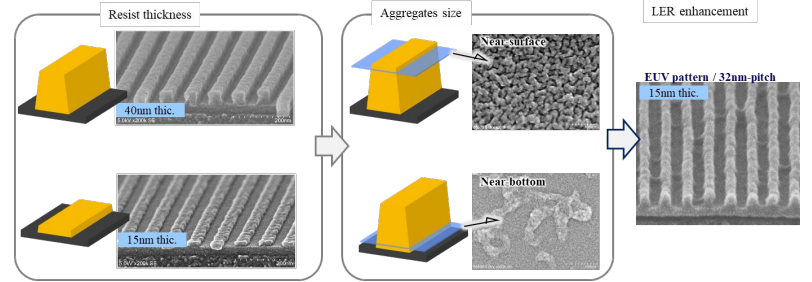

Fig. 17. Assumption of induction factor of LER with resist film thickness.

\subsection{Resist behavior in opened space region}

The most serious problems today are residual types of defects such as missing holes and bridge lines, and it is really waiting for a solution. To find a solution to minimize defects, the behavior of the resist was investigated by the polymer dynamics aspects, similarly as polymer clusters observation. The focal area of observation is the very low dose state stage before the formation of dotted polymer aggregates, which corresponds to the unexposed open space area of the resist pattern. In this work, as shown in Figure. 18, four types of lower layers with different surface free energies were prepared. The two key factors that make up the surface free energy, the dispersion and polarity components, have changed in the bottom four layers (UL-A to D).

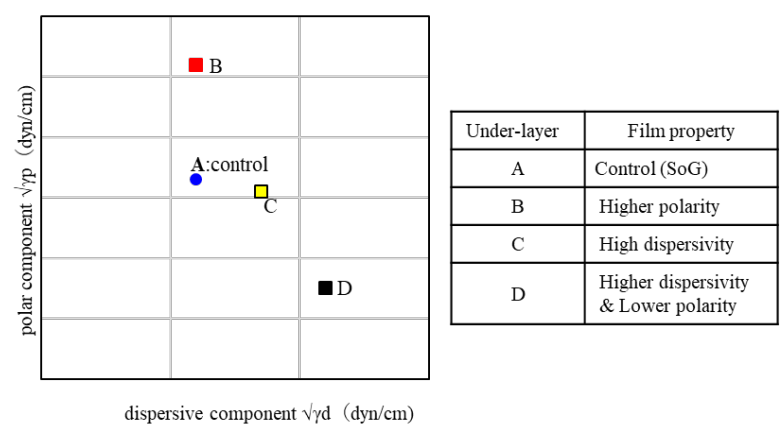

Fig. 18. $\gamma$ value elements of UL material.

The observation criteria were SEM image acquisition and roughness measurement with an atomic force microscope (AFM). Figure 19 shows an image comparison of defects. UL-A and $\mathrm{C}$ defects are large, and UL-B and D are significantly smaller. As previously reported in SPIE [4], UL-B has the advantage of reducing defects over UL-A. Therefore, this result may represent other evidence of defect mitigation capabilities. In addition, the analysis results by X-ray photoelectron spectroscopy (XPS) are also shown in Figure 20. This is a depth profile of the qualitative analysis results comparing the cases of 


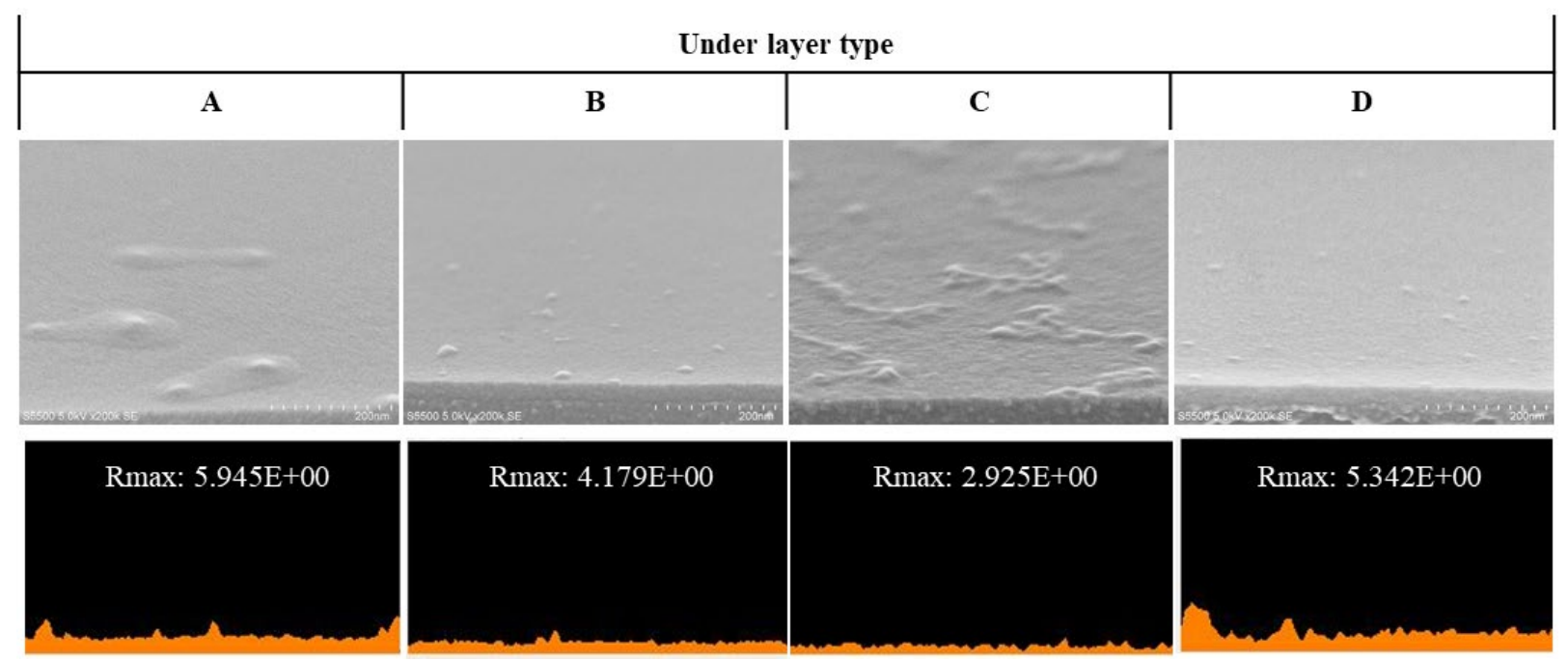

Fig. 19. Resist residue SEM image and AFM profile.
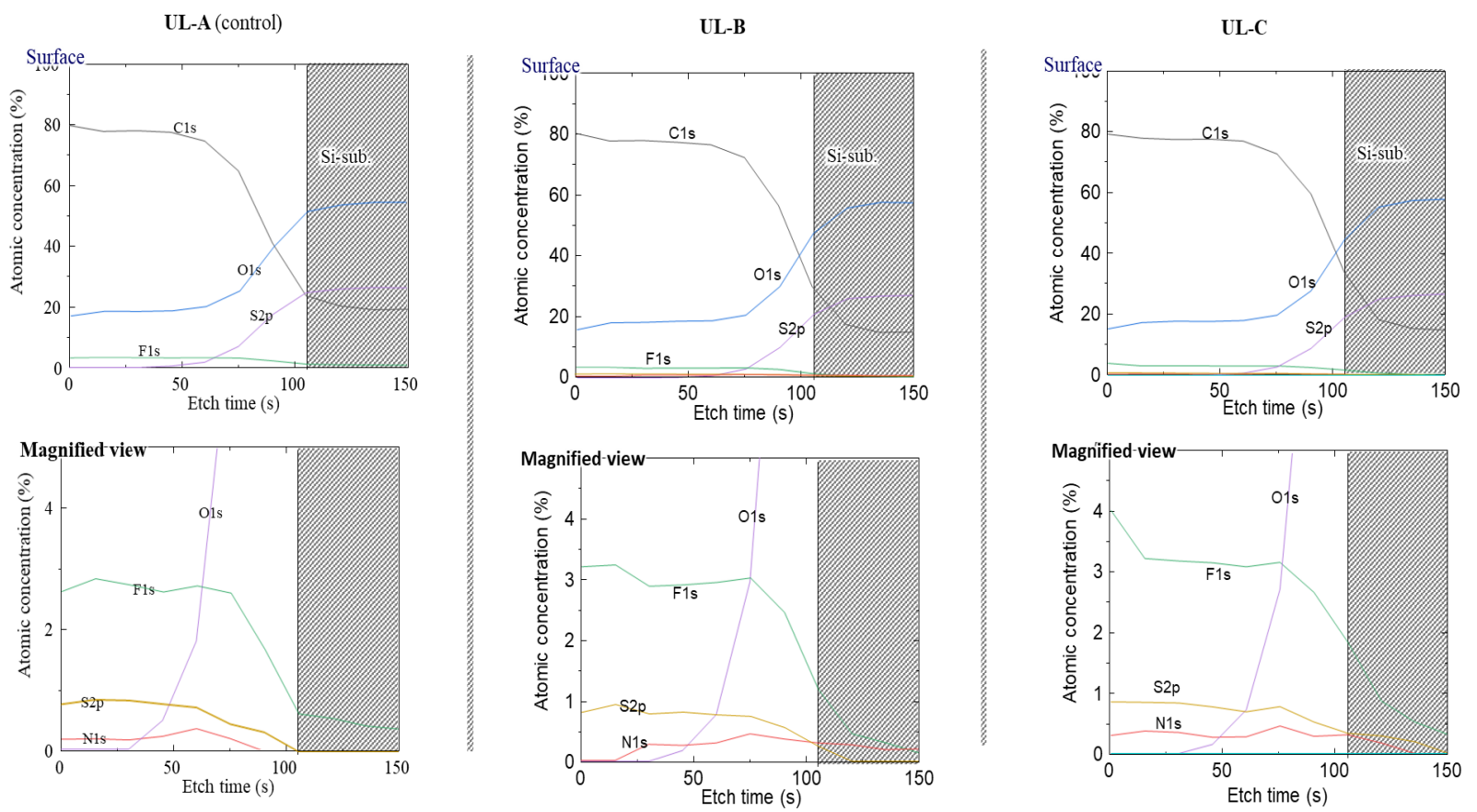

Fig. 20. XPS analytical result comparison in 3 kind of under layer material.

three different under layers. Target elements are sulfur, fluorine, nitrogen and others found in PAG or quencher derivatives and other additives. The additives never move and do not appear to change the surface-to-bottom distribution of the resist under several different underlayer conditions. And it can be understood that the properties of the residue are not due to the influence of the resist additive.

Table 7 summarizes the relevant factors to reduce the number of defects. From this table, it can be pointed out that the adhesive work may be the most effective indicator of affecting defects. The condition with lower adhesive work appears to cause desorption of residues from the substrate.
In addition, the material of the underlayer is also noteworthy. Many defects were found on composite type underlayer but reduced on single component one. The atomic size is microscopic and cannot be measured at present, however, it looks that the elemental formation of the underlayer material was relatively uniform compared to the composite type. Several preferred methods for surface condition analysis may be proposed in the future to replace current contact angle measurement methods.

\section{Conclusion}

In this paper, several types of randomness were visualized and quantified, targeting any stochastic 
Table 7. Summarized comparison of defect density and impacted factor.

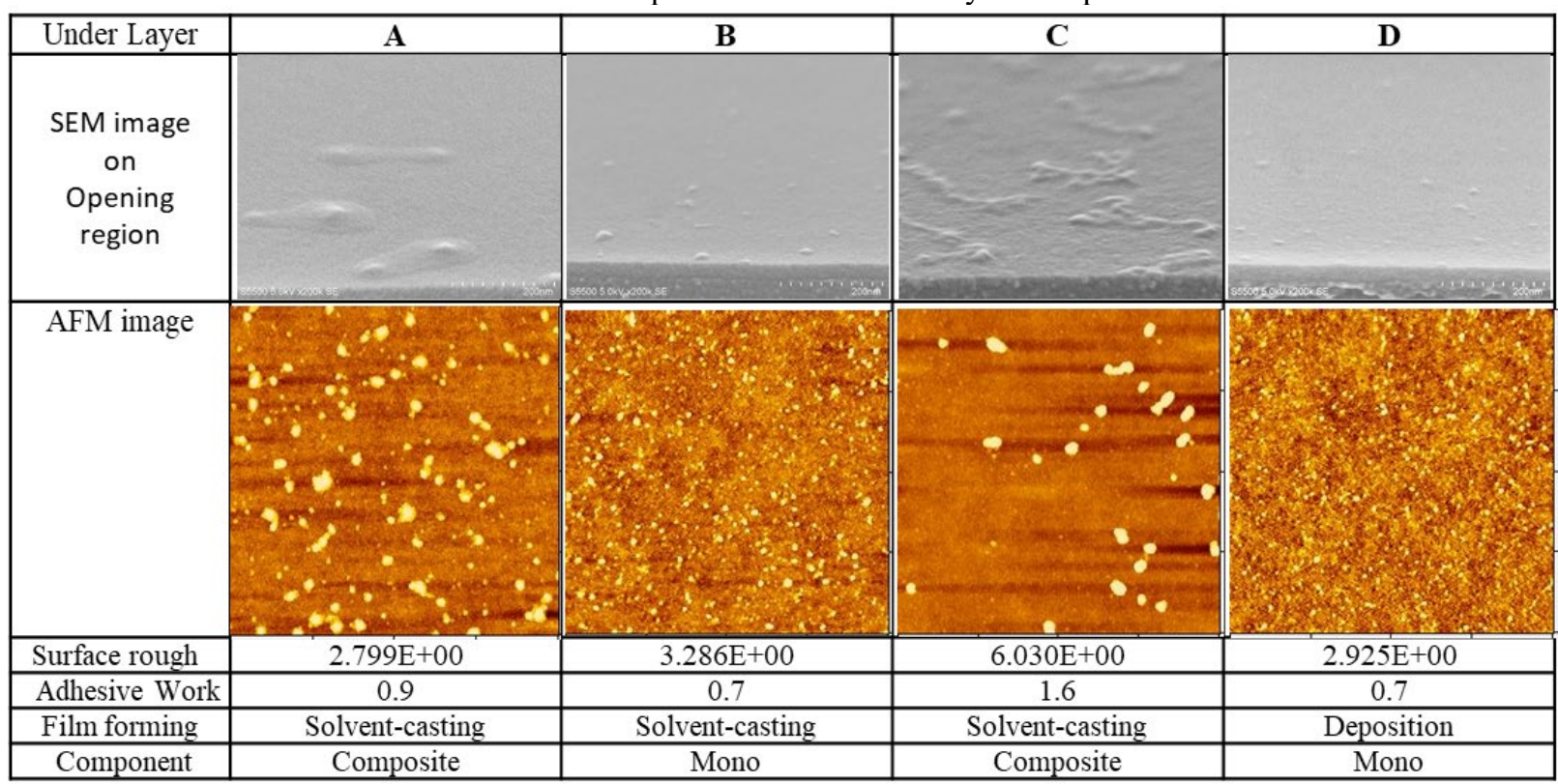

phenomena caused by local variation and residual types defects in the EUV process. The points regarding LER are as follows.

$>$ Resist pattern consists with pixelated polymer aggregates.

$>$ Its pixel size was approximately $12 \mathrm{~nm}$.

$>$ It might correspond to resolution limit.

It can also be said that polymer aggregate must be related with defect density. Important factor may be polymer design in resist synthesis and surface condition of underlayer. Cross-section SEMs were primarily used in this work, but non-destructive methodologies with short turnaround times must be considered in the future.

\section{References}

1. Kozawa, T., and Tagawa, S., "Radiation chemistry in chemically amplified resists", Jap. J. Appl. Phys. 49 (2010)

2. Tarutani, S., Tsubaki, H., and Kanna, S., "Development of materials and processes for negative tone development toward 32-nm node 193-nm immersion double-patterning process", Proc. SPIE 7273, 72730C (2009)

3. Cutler, C. A., Mackevich, J. F., Li, J., O'Connell, D. J., Cardinale, G. F., and Brainard, R. L., "Effect of Polymer Molecular Weight on AFM Polymer Aggregate Size and LER of EUV Resists", Proc. of SPIE Vol. 5037 (2003)
4. Yaegashi, H., Hara, A., Okada, S., and Shimura, S., "Explorations of missing hole defect in EUV patterning", proc. of SPIE 11326-13 (2020)

5. Hohle, C. K., Heckmann, N., Sebald, M., Markert, M., Stepanenko, N., Houlihan, F. M., Romano, A. R., Sakamuri, R., Rentkiewicz, D., and Dammel, R. R., "Surface roughness investigation of 157- and 193-nm polymer platforms using different etch conditions", J. Microlith., Microfab., Microsyst. 4(4), 043009 (2005)

6. Oehrlein, G. S., Phaneuf, R. J., and Graves, D. B., "Plasma-polymer interactions: A review of progress in understanding polymer resist mask durability during plasma etching for nanoscale fabrication", J. Vac. Sci. Technol. B 29 (2011)

7. Takeuchi, T., Corbella, C., Kreul, S. G., Keudell, A. V., Ishikawa, K., Kondo, H., Takeda, K., Sekine, M., and Hori, M., "Development of the sputtering yields of ArF photoresist after the onset of argon ion bombardment", Journal of Applied Physics 113, 014306 (2013)

8. Ito, H., Willson, C. G., Frechet, J. H. J., "New UV resists with negative or positive tone", Symposium on VLSI Technology (1982)

9. Yaegashi, H., Koike, K., "Reconsiderations of LER characterization", proc. of SPIE, 10586-8 (2018) 